\title{
Single-trial free recall from temporal search sets in long-term memory
}

\author{
RALPH E. GEISELMAN \\ Ohio University, Athens, Ohio 45701
}

\begin{abstract}
The notion that the primacy effect, which is found in single-trial free-recall experiments, is partly a function of a selective-search component (Shiffrin, 1970) is contingent upon the ability of subjects to retrieve information via a distinctive temporal cue. The beginning of a list may be such a cue which defines a restricted temporal search set within a list as a whole. To test this theory, a second list-half primacy effect was generated in some 26 "unrelated" words lists by associating one color with each word in the first list half and another color with each word in the second list half. As predicted by the two-process theory, retrieval of the words which were presented around the color shift was differentially facilitated as measured by the difference between the probabilities of recall and recognition at each serial position and as compared to that of lists where the color codes were randomly presented.
\end{abstract}

Shiffrin (1970) has suggested that the primacy effect which is found in free-recall serial-position curves may be due in part to a selective-search mechanism, rather than solely to a selective-rehearsal mechanism. Theoretically, either mechanism could account for the effect. Under the selective-rehearsal conception, the first few items presented in a list enter an unfilled memory buffer in short-term store and therefore receive more rehearsal effort than items presented later in the list. The more that an item is effectively rehearsed, the greater the probability of the item gaining storage in long-term store (Atkinson \& Shiffrin, 1968; Shiffrin \& Atkinson, 1969). Under the selective-search conception (Shiffrin, 1970), the beginning of a list provides a distinctive cue which defines a restricted, temporal search set within the list as a whole. The probability of drawing a particular primacy area item from the primacy search set is proposed to be equal to its strength in memory divided by the sum of the strengths of all of the items in the primacy search set. Since the nonprimacy-area items can only be drawn from the search set composed of the list as a whole, a primacy effect is predicted independently of differential item storage strength due to selective rehearsal. Shiffrin does not postulate how or when the primacy search set is complete; but since it is said to be defined temporally, an item closer to the beginning of the list would have a greater probability of being in the search set than would an item presented later in the list. Bellezza, Andrasik, and Lewis (Note 1) have suggested that the primacy search set may be complete when the subject loses track of how many items have been presented.

Shiffrin (1970) has pointed out that since primacy effects have been found in recognition serial-position curves (Norman \& Waugh, 1968), a mechanism of

Requests for reprints should be sent to Ralph E. Geiselman, Department of Psychology, Ohio University, Athens, Ohio 45701. selective rehearsal cannot be discounted completely. If all items in a list have the same strength in memory, then tasks where the role of search processes is essentially absent should yield no primacy effect. Whether selective search is a factor in producing the primacy effect is an open question. Restricted search sets can be temporally defined (Kay \& Poulton, 1951), but temporal definition processes alone may merely promote selective rehearsal of distinctive items at the expense of other items. Most studies of the von Restorff effect report that lists with isolated items are learned no better than corresponding control lists (Wallace, 1965). Selective-search mechanisms would result in better recall of lists with isolated items. Another possibility is that both mechanisms, search and rehearsal, are contributors to the resulting primacy effect to some degree. This paper addresses itself to the storage plus search or "two-process" possibility.

One approach in assessing the two-process theory is to prevent selective rehearsal during list presentation. If the primacy effect is solely the result of selective rehearsal, then no primacy effect should be found. Bjork and Whitten (1974) presented a series of word pairs to subjects with each pair being preceded and followed by interpolated activity. Though the subjects were instructed to rehearse each word pair individually, a significant primacy-area effect was still observed for the list as a whole. The authors suggested that either a transient retrieval process or output interference could account for the effect. Glanzer and Meinzer (1967), using a procedure similar to that of Bjork and Whitten, have also shown that the primacy effect is not totally eliminated by instructions to only rehearse the item that has just been presented during each unfilled interitem interval.

Another means of assessing the possibility of a dual-process contribution to the intralist primacy effect, and one which was employed in the present investigation 
of the problem, is to provide a second temporal cue in the middle of a list to define a second list-half primacy effect (Bellezza, et al., 1973). If a selective-rehearsal mechanism were the sole contributor to the second list-half primacy effect, then there would be no increase in the free recall of the list as a whole. The first few items of the second half of the list would receive selective rehearsal only at the expense of the last few items of the first list half. A problem with this type of investigation is that to insure the temporal definition of the second list-half primacy set, the experimenter must necessarily increase the subject's information load. For example, consider the procedure which was used in the present investigation. One color code was associated with each word in the first half of a list and another color code with each word in the second list half. Instructions to remember the color with each word insured two temporal cues (i.e., the beginning of the list and the color-code changeover) to define a primacy set in each list half, but the subjects' information loads were simultaneously increased. Any retrieval facilitation due to searching the second restricted memory set was possibly offset by the increased information load. In order to illustrate how this confound was eliminated, it is necessary to focus on a comparison of recall and recognition.

At a fixed presentation time per list item, a subject can process list information for purposes of delayed recognition (transfer of new information to long-term store) or delayed recall (coding of new information and transfer to long-term store) depending on when and what a subject learns or is told about the ensuing delayed test (Bernbach, 1973; Carey \& Lockhart, 1973; Craik \& Watkins, 1973; Loftus, 1971; Tversky, 1973). In the present investigation, the subjects were told that half of a series of word lists would be tested for delayed recall and half for delayed recognition. Let $W_{1}$ represent the amount of list presentation time that a subject uses for purposes of encoding and storage of a list of words for later recall from long-term store; and let $W_{2}$ represent the amount of list presentation time that the same subject uses for purposes of encoding and storage of the same list for later recognition from long-term store. Let $T$ be the total list presentation time which is equal to $W_{1}+W_{2}$. The ratio of $W_{1}$ to $W_{2}$ is determined by the subject's processing strategy at list presentation and therefore by all factors influencing the subject's strategy such as instructions from the experimenter and possibly $\mathrm{T}$. With the addition of item color codes to be processed, $T$ is equal to $W_{1}+W_{2}+C T$; where $C T$ is the amount of list presentation time used for purposes of color tagging the list items. $C T$ reduces $W_{1}$ and/or $W_{2}$ which in turn lowers the probability of recall, $p(\operatorname{Re})$, and/or the probability of recognition $p(R o)$. To account for CT, the first task was to plot $p(\operatorname{Re})$ vs. $p(R o)$ for various presentation times when no simultaneous color-code processing was demanded. The resulting curve was then used, along with $\mathrm{p}(\mathrm{Ro})$ when blocked, binary color-code processing was introduced, to predict $p(\operatorname{Re})$ with blocked, binary color-code processing. Thus, a second temporal cue was supplied in the form of a midlist color shift with the additional memory load to be taken into account by the predictor curve. If the obtained $\mathrm{p}(\mathrm{Re})$ with blocked color coding were found to be greater than that as predicted, then defining a primacy set in each list half could be said to promote more efficient retrieval than a single primary set in the list as a whole. Since presenting the color codes in a random or unblocked fashion would define only one restricted, temporal search set (i.e., the beginning of the list), $\mathrm{p}(\mathrm{Re})$ with unblocked color coding should be statistically the same as predicted from the predictor curve and $\mathrm{p}(\mathrm{Ro})$ with unblocked color coding. This equality would not be observed if the introduction of the color codes caused the subjects to change their $\mathrm{W}_{1} / \mathrm{W}_{2}$ ratios in a fashion not predicted by the predictor curve.

Mandle r (1972) failed to find subjective organizational effects on "list-specific" recognition (interlist confusions). Therefore, the distractors in the recognition tests were taken from previously presented lists so that $p(R o)$ could be used as a predictor of $p(R e)$ without introducing a confound due to differences in subjective organizational activity in the blocked and unblocked color-code conditions.

\section{OBTAINING THE PREDICTOR CURVE}

\section{Method}

Subjects. The subjects were 32 undergraduates from various psychology courses at Ohio University who volunteered to serve in a psychology experiment for course credit.

Materials and apparatus. Twelve 26-word lists were presented to each subject using a Lafayette, IBM memory drum. The words used had a Thorndike and Lorge count of between 10 and 40 occurrences per million words. Two different sets of 12 lists were used.

Procedure. The subjects were told to say each word aloud as it was presented and that each of the list presentations would be followed by a $20-\mathrm{sec}$ interpolated task. The task was to count backward from a given 3-digit number by sevens. One count was required every $2 \mathrm{sec}$. The subjects were also told that half of the subsequent retention tests would be free-recall and half would be four-al ternative forced-choice recognition. The recognition tests were composed of 13 list items randomized with respect to serial position, each matched with three distractors of the same serial position, one from each of the three just preceding lists. The first practice recognition test necessarily had two distractors which had not been previously presented. The nature of each test was determined by the subject by turning a page in a response booklet after each interpoloated task. The first four trials (lists) were practice trials with presentation times of $3.00 \mathrm{sec}$ per word, $2.25 \mathrm{sec}$ per word, $1.50 \mathrm{sec}$ per word, and $0.75 \mathrm{sec}$ per word. Two of the tests were recognition and two recall. The remaining eight lists were scored (four recall and four recognition tests) with two instances of each presentation time (one with recall, one with recognition). The order of occurrence of the presentation times and the type of test was randomized 
within and between subjects. The only information that was given to the subjects before a trial was whether the next presentation time would be slower or faster than the previous one. The subjects were given 1-1/2 min to comple te each test.

Design. The data matrix collapsed across the subjects was a 4 by 2 . The factors were presentation time by type of test. The obtained recognition data were linearly corrected for guessing: $p(c)=c+(1-c) g$, where $g=.25$.

\section{Results}

The results of the predictor-curve investigation are displayed in Table 1. An analysis of variance showed a significant test type effect $[\mathrm{F}(1,31)=181.1, \mathrm{p}<.001$, $\left.\mathrm{MS}_{\mathrm{e}}=19.1\right]$, a significant presentation time effect $\left[\mathrm{F}(3,93) 98.9, \mathrm{p}<.001, \mathrm{MS}_{\mathrm{e}}=2.1\right]$, and a significant Test Type by Presentation Time interaction effect $\left[\mathrm{F}(3,93)=11.3, \mathrm{p}<.01, \mathrm{MS}_{\mathrm{e}}=2.3\right]$. The recall and recognition retention curves as a function of presentation time are clearly both linear for the range of presentation times observed. Further, the slope of the recognition-retention function is exactly twice as large as that of the recall-retention function.

The probability of recall was plotted vs. the probability of recognition for each presentation time, and a least squares analysis of the four data points produced the equation, $\mathrm{Y}=-0.12+0.48(\mathrm{X})$. This equation was then used to obtain a predicted value of recall $\left(Y^{\prime}\right)$ from each subject's obtained value of recognition $(\mathrm{X})$. The four predicted mean values of recall were $1.8,2.8,3.9$, and 5.6. A 2 by 4 ANOVA was conducted with recall (obtained and predicted) and presentation time as the factors. The recall effect was nonsignificant $\left[\mathrm{F}(1,31)=.04, \mathrm{p}>.99, \mathrm{MS}_{\mathrm{e}}=1.37\right]$ as was the Presentation Time by Recall interaction effect. Though the obtained curve is slightly curvilinear, the restricted range involved permitted the unaltered linear representation to be used as the predictor curve.

\section{PROVIDING A SECOND TEMPORAL CUE}

\section{Method}

Subjects. The subjects were 47 undergraduates from the subject pool at Ohio University.

Materials and apparatus. The same two sets of word lists and the same apparatus as was used in obtaining the predictor curve were employed.

Procedure. The first four lists were again used as practice lists but all lists were presented with a presentation time of $3 \mathrm{sec}$ per word. The subjects were told that $50 \%$ of all lists and in terpoloated tasks would be followed by recall tests and half by recognition tests. The fifth and sixth trial tests were with noncolor-coded words, one recall and one recognition. This condition is designated the (I), remember items only, condition. The next six trials were color-coding trials with color dots, red or black, placed beside each word. There were 13 red dots and 13 black dots in each list. The subjects were told to remember each color code with each word and that, "a correctly remembered word with an incorrect color code will be scored as incorrect." The experimenter did not, in fact, score the color codes for purposes of the analysis because doing so might have injected a color-code discriminability confound into the word-retention results. Either the seventh through the ninth or tenth through
Table 1

Mean Number of Words Recognized and Recalled as a Function of Presentation Time

\begin{tabular}{ccc}
\hline \multirow{2}{*}{$\begin{array}{c}\text { Presentation } \\
\text { Time* }\end{array}$} & \multicolumn{2}{c}{ Type of Test } \\
\cline { 2 - 3 } & Recognition** & Recall \\
\hline .75 & 10.3 & 1.6 \\
1.50 & 12.5 & 3.0 \\
2.25 & 14.8 & 4.1 \\
3.00 & 18.3 & 5.5 \\
\hline
\end{tabular}

* Presentation time is in seconds.

**The recognition data are linearly corrected for guessing.

the twelfth trials had the color dots arranged in blocks, all red first or all black first. This condition is designated the $(\mathrm{I}+\mathrm{C} \mid \mathrm{BL})$, remember items plus color codes given color-code blocking, condition. The other three trials had the color dots arranged in a random or unblocked fashion, (I + C|UNBL). Before each set of three color-coding trials, the subjects were told the nature of the color-code arrangement and that they must say each word followed by its color code as each word was presented. Only the last two lists in each of these two sets were scored, one for recall and one for recognition.

Under the (I) condition, there should be no significant difference between predicted recall $\left(\mathrm{Y}^{\prime}\right)$ and obtained recall $(\mathrm{Y})$ unless there are significant group differences in memory performance. Under the (I + C|UNBL) condition, there should be no significant difference between $\mathrm{Y}^{\prime}$ and $\mathrm{Y}$ obtained unless the introduction of the unblocked color codes alters the $W_{1} / W_{2}$ ratio in a manner not predicted by the predictor curve. The recall and recognition tests were given equally often, and therefore the $(\mathrm{I}+\mathrm{C})$ time-allocation strategies at any value of $\left(\mathrm{V}_{1}+\mathrm{W}_{2}\right)$ should correspond to those obtained from the predictor curve. Under the $(\mathrm{I}+\mathrm{C} \mid \mathrm{BL})$ condition, the $\mathrm{Y}$ obtained would be greater than $\mathrm{Y}^{\prime}$ if list-half primacy sets promote more efficient retrieval than a single list primacy effect. The $Y$ obtained would be statistically the same as $Y^{\prime}$ if the list-half primacy effects are due solely to selective rehearsal. An additional prediction was that $\mathrm{p}(\mathrm{Re})$ and $\mathrm{p}(\mathrm{Ro})$ would be largest for the (I) condition, and next largest for the $(I+C \mid B L)$ condition, and smallest for the $(I+C \mid U N B L)$ condition. This prediction follows from the amount of information to be processed. The $(\mathrm{I}+\mathrm{C} / \mathrm{BL})$ condition has less uncertainty associated with each item color code than does the $(\mathrm{I}+\mathrm{C} \mid \mathrm{UNBL})$ condition.

\section{Results}

After observing a few subjects with the procedure outlined above, it was noticed that many of the subjects were completely inaccurate in their color coding of words correctly recalled and recognized. This occurred even though the instructions explicitly stated that a correctly recalled word would be scored incorrect unless the correct color code was also remembered. The failure to process the color-code information by some subjects defeated the experimenter's purpose of having color codes. Therefore, a chi-square test was done on each subject's data to determine whether each subject was processing color significantly better than chance, $\mathrm{p}<.05$. Chance for each individual subject was taken to be one-half of the correctly recalled words plus one-half of the correctly recognized words on the last six trials, three recall tests plus three recognition tests. Forty-seven 
Table 2

Mean Number of Words Recognized and Recalled as a Function of Presentation Condition

\begin{tabular}{ccc}
\hline & \multicolumn{2}{c}{ Type of Test } \\
\cline { 2 - 3 } Condition* & Recognition** & Recall \\
\hline (I) & 18.0 & 5.5 \\
(I + C / BL) & 14.3 & 5.0 \\
(I + C I UNBL) & 13.3 & 3.4 \\
\hline
\end{tabular}

$*(I)=$ items only, $(I+C \mid B L)=$ items plus color codes given a blocked input order, and $(I+C \mid U N B L)=$ items plus color codes given an unblocked input order.

**The recognition data are linearly corrected for guessing.

subjects were tested in the procedure before 32 subjects were found who processed color significantly better than chance. The average number of correctly recalled words plus correctly recognized words on the last six trials was 56.0 for the color processing subjects and 67.2 for the other subjects. If the levels of recall plus recognition in the (I) condition (Trials 5 and 6) for the latter subjects is taken times three, a comparable figure of 69.5 is obtained. These subjects were essentially performing six trials of the (I) condition rather than processing the color codes. The mean chi square for the color processing subjects was $13.0, \mathrm{p}<.001$, and was 1.21 , $p>.20$, for the other subjects. This result may have been due to the difficulty of the present task. It was desirable to eliminate these noncolor-coding subjects because they may not have received the second temporal cue. It is also perfectly acceptable in the present situation to eliminate these subjects because an ability check between the experimental group and the predictor-curve group is built into the design, namely the (I) condition at the $3-\mathrm{sec}$ presentation rate. If the experimental and predictor-curve groups differ in this condition, the two groups would then be uncomparable. This process of objectively removing subjects from the data matrix is not a new one. Reitman, Malin, Bjork, and Higman (1973) eliminated $61 \%$ of their subjects for not accurately following instructions on the basis of a postexperimental interview.

The mean number of words recalled and recognized in the three presentation conditions for the 32 acceptable subjects are shown in Table 2 . These values were obtained without regard to correct color coding, and the recognition data is corrected for guessing as outlined earlier. Inspection of the (I) condition results shows that the elimination of the subjects who failed to process the color codes did not produce an experimental group of superior memory ability.

The predictor-curve equation was used to obtain a predicted value of recall $\left(\mathrm{Y}^{\prime}\right)$ in each of the three conditions for each acceptable subject. A 3 by 2 ANOVA was then conducted with condition and recall ( $\mathrm{Y}^{\prime}, \mathrm{Y}$ obtained) as the factors. The Condition by Recall interaction effect was significant $[F(2,62)=4.5$, $\left.\mathrm{p}<.05, \mathrm{MS}_{\mathrm{e}}=1.7\right]$. A Cicchetti (1972) test showed that $Y^{\prime}$ was significantly different from $Y$ obtained for the $(I+C \mid B L)$ condition only, $p<.01$. Figure 1 is $p(R e)$ plotted vs. p(Ro) and shows the predictor curve as well as three points representing the three presentation conditions. Each data point in the figure represents 32 recall and 32 recognition observations.

The lower portion of Figure 2 shows the recall serial-position (SP) curves for the three conditions. A trend analysis was conducted on each curve collapsed into six SP groups. The (I) condition curve has a significant linear component $[F(1,155)=14.4, p<.01$, $\left.\mathrm{MS}_{\mathrm{e}}=.7\right]$, representing a significant primacy effect. The $(\mathrm{I}+\mathrm{C} \mid \mathrm{UNBL})$ condition also has a significant linear component $\left[\mathrm{F}(1,155)=24.7, \mathrm{p}<.01, \mathrm{MS}_{\mathrm{e}}=.6\right]$. Likewise, the $(\mathrm{I}+\mathrm{C} \mid \mathrm{BL})$ condition has a significant linear component $\left[F(1,155)=13.9, p<.01, M_{e}=.6\right]$, but also a significant cubic component $[F(1,155)=24.3$, $p<.01]$. The cubic component signifies the significant second list half primacy effect. Bellezza et al. (1973) found a similar second-area effect by associating a " 1 " with each word in the first list half and a " 2 " with each word in the second list half. Also included in Figure 2 is an output percentile curve for each condition, shown in the upper half of the figure. The output percentile was developed by Bjork and Whitten (1974) as an unbiased estimate of output position. Each correctly recalled word has its output position divided by the total number of words that the subject recalled on that trial. This number is then multiplied by 100 to obtain a percentile score. The data points shown in Figure 2 represent mean output percentile scores for six different SP ranges. As was found by Bjork and Whitten, using a different presentation procedure, the output curves are nearly mirror images of the recall SP curves. In the $(I+C \mid B L)$ condition, an average subject first gave the first primacy-area words, then the words immediately following the color shift.

Figure 3 shows the three recognition SP curves for the three presentation conditions. Three trend analyses showed that the same trends exist in the recognition data as in the recall data, though the $F$ values are much

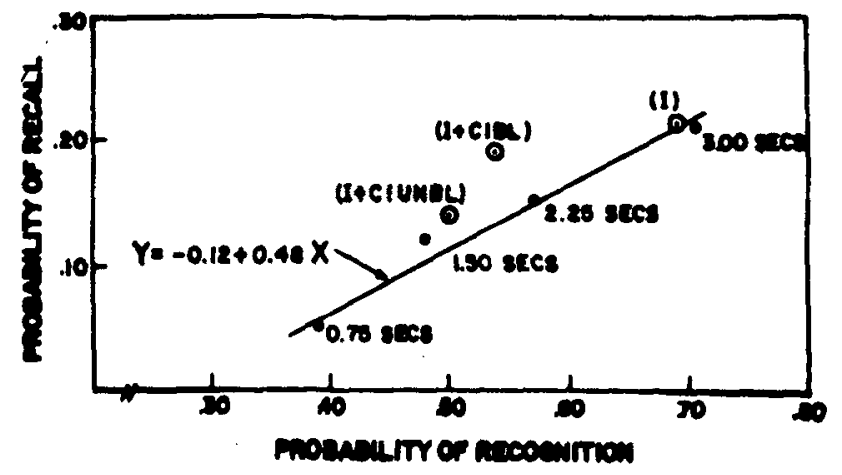

Figure 1. The recall-recognition predictor curve and three points representing the three presentation conditions. 
smaller. The $\mathrm{F}$ values for the linear trends for the (I), (I + ClUNBL), and (I+C|BL) conditions respectively are $\left[\mathrm{F}(1,155)=4.7, \mathrm{p}<.05, \mathrm{MS}_{\mathrm{e}}=1.3\right],[\mathrm{F}(1,155)=5.9$, $\left.\mathrm{p}<.05, \mathrm{MS}_{\mathrm{e}}=.8\right]$, and $[\mathrm{F}(1,155)=7.8, \mathrm{p}<.01$, $\left.\mathrm{MS}_{\mathrm{e}}=.8\right]$. The cubic trend in the $(\mathrm{I}+\mathrm{C} \mid \mathrm{BL})$ condition was again significant $[F(1,55)=16.0, \mathrm{p}<.01]$, indicating that the second list-half primacy effect was due in large part to differential storage strength, the result of differential selective rehearsal.

Since the $\mathrm{p}(\mathrm{Re})$ obtained in the $(\mathrm{I}+\mathrm{C} \mid \mathrm{BL})$ condition is larger than predicted, the number of list words which were recognized but not recalled in the $(I+C \mid B L)$ condition is smaller than would be the case in the (I+C|UNBL) condition with the same $\mathrm{p}(\mathrm{Ro})$. To determine which SPs received the additional retrieval facilitation, $p(R e)$ was subtracted from $p(R o)$ at each $S P$ in the $(I+C \mid B L)$ condition and in the $(I+C \mid U N B L)$ condition. $[p(R o)-p(R e)]$ represents the probability of a word being recognized but not recalled. The results are plotted in Figure 4 with the 26 SPs collapsed into six position groups. There is an apparent difference in the shapes of the two curves. The retrieval facilitation promoted by the second list-half primacy set was restricted to the middle portion of the SP curve, to those words immediately preceding and following the color shift. Even though there were negative recognition and recall recency effects in SPs 9-13, and these words were relatively the last to be given as output in free recall, their accessibility was facilitated by their temporal proximity to the second distinctive temporal cue. The fact that the words immediately preceding the color shift were of the first color, as well as their low memory strength, may have affected their probability of being drawn earlier from the second restricted search set.

\section{DISCUSSION}

The $p(\operatorname{Re})$ obtained in the $(\mathrm{I}+\mathrm{C} \mid \mathrm{BL})$ condition cannot be explained by a mechanism of selective rehearsal alone. Even though $\mathrm{p}(\mathrm{Re})$ in the $(I+\mathrm{C} \mid \mathrm{BL})$ condition was slightly less than that in the (I) condition, forming list-half primacy sets facilitated retrieval. It is unlikely that the $W_{1} / W_{2}$ ratio was altered in a manner not predicted by the predictor curve because the same subjects had the opportunity to increase this ratio in the (I + ClUNBL) condition where CT was even larger. Further, it would have to be argued that the subjects became unpredictably concerned about coding for later recall during the presentation of the middle of the list only. In this regard, retrieval facilitation similar to that in the $(\mathrm{I}+\mathrm{C} / \mathrm{BL})$ condition has been observed by the author around distinctive events when subjects could not possibly anticipate the temporal position of the events. As an example, Figure 5 shows $[p(\operatorname{Ro})-p(\operatorname{Re})]$ plotted vs. SP from an experiment in which the subjects were presented with lists of unrelated words, some containing

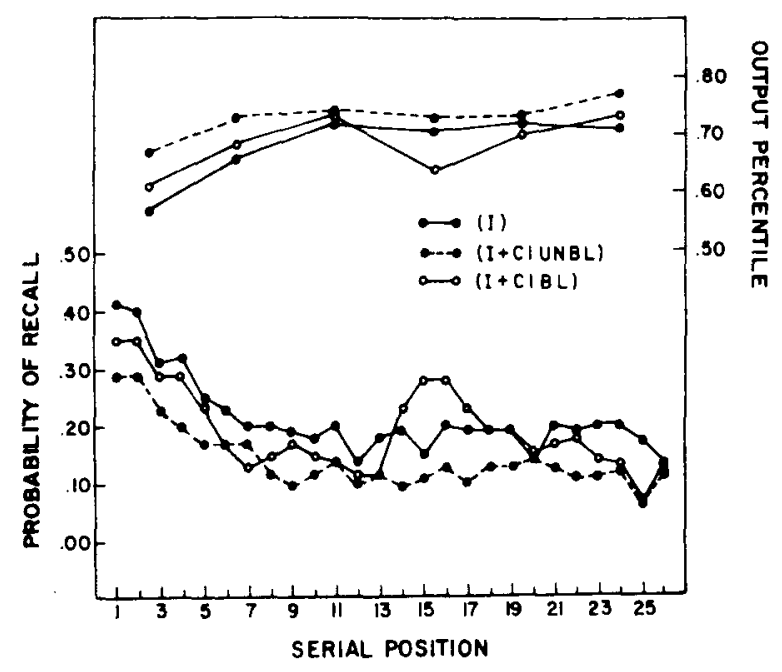

Figure 2. Recall serial-position curves and output-percentile curves for the three presentation conditions.

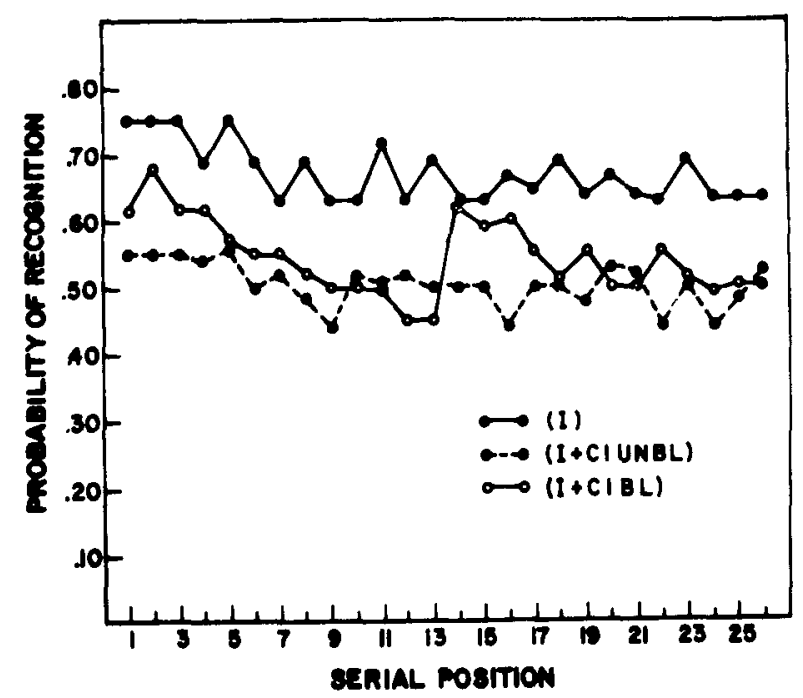

Figure 3. Recognition serial-position curves for the three presentation conditions.

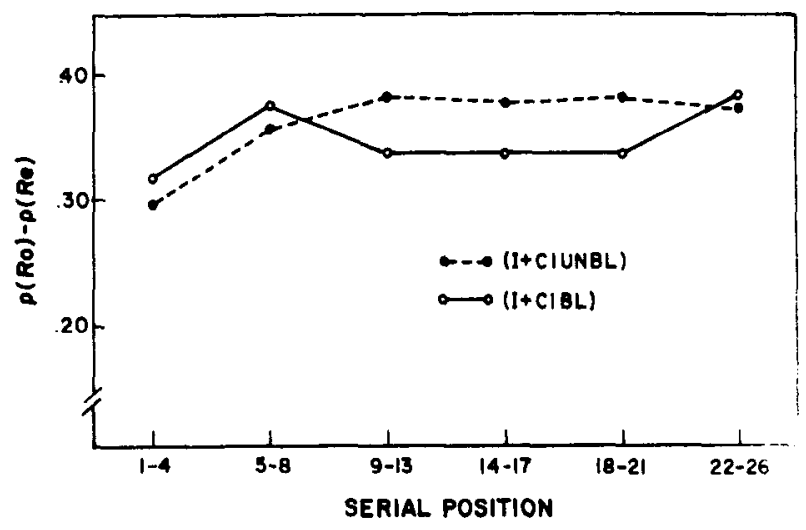

Figure 4. The probability of recognition minus the probability of recall as a function of serial position for the $(I+\mathrm{C} \mid \mathrm{UNBL})$ and $(I+C \mid B L)$ presentation conditions. 


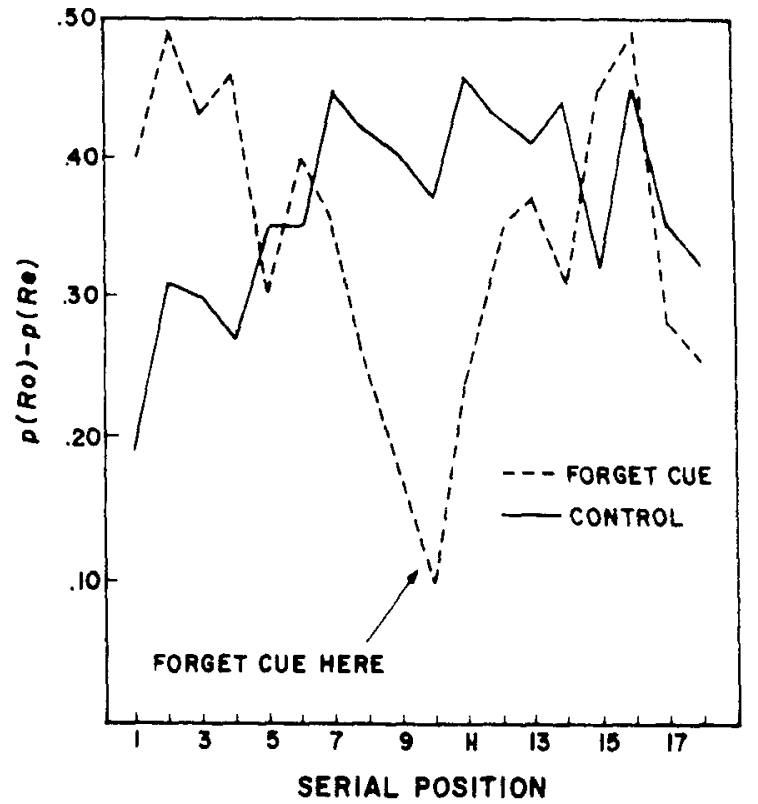

Figure 5. The probability of recognition minus the probability of recall as a function of serial position for lists with a forget cue and for control lists.

a cue to forget the previously presented words in the list. The cue appeared at a different position in different lists, if it appeared at all. The data in Figure 5 are taken from the last trial which involved a surprise test on the entire list. The last list contained 18 words and, for half of the subjects, a forget cue in the middle of the list. The distractors for yes-no recognition tests were taken from the intrusion errors given by other subjects in corresponding free-recall conditions. As shown in Figure 5, retrieval was most efficient for the forget-cue group just before and just after the forget cue. These subjects could not have anticipated the position or even the occurrence of the cue.

The negative midlist recency effects shown in Figures 2 and 3 are consonant with those of Bruce and Papay (1970) which they obtained by positioning a forget cue in the middle of a list. But their argument that the negative recency effects are evidence against a partial, selective-retrieval explanation of their second list-half primacy effect is questionable. Bruce and Papay scaled recall and recognition performance, but not between SP retrieval efficiency. A relation similar to that shown in Figure 5 can be obtained by subtracting Bruce and Papay's (1970, Figure 4) forget-cue and control recall curves from their corresponding recognition curves.

An aspect of Shiffrin's (1970) search-set formalization supported by Figure 4 which seems unclear intuitively, is that if a restricted search set facilitates recall of the items within the search set, then why isn't recall of the other list items facilitated as well? In an unmodified word list, if the primacy-area words are designated as members of the search set $P$, then the other list items should be members of the restricted set NP or "not P." Figure 4 implies that traditional set theory does not necessarily apply to memory search processes. The search sets $\mathbf{P}_{\mathbf{1}}, \mathbf{P}_{\mathbf{2}}$, and $\mathrm{L}$ ("list") were formed but not $\mathrm{NP}_{1}$ or $\mathrm{NP}_{2}$. The subjects either could not search nondistinct list areas as restricted entities or they did not think of doing so.

In any event, the present results provide promising support for the theory that two memory mechanisms are responsible for the primacy effect rather than one. The exact nature of the selective-search contribution is still rather hazy and many questions remain for future research. For example, how are temporal, primacy search sets completed? Possibly the distinctive cue "tags" those items which are in short-term store buffer at the time of the cue's occurrence plus the items which fill the buffer anew immediately after the cue. Another important question is whether temporal search sets can be "smeared" by subjective reorganization of the input SPs. Bjork and Whitten (1974) cautioned that the primacy effect which they obtained after minimizing selective rehearsal may reflect different processes than do typical primacy effects. In unmodified free recall procedures, the subjects are free to organize the list items in idyosyncratic fashions and consequently, the nominal input positions may become smeared in time. Research on overt rehearsal characteristics by Melton and Glenburg, mentioned by Bjork and Whitten, suggests that an item usually does lose its temporal input position via further cognitive processing. The present procedure of forcing the subjects to say each word and color code aloud with a presentation time of three seconds per word may have severely limited this idyosyncratic reorganization. Consequently, future research may show that the magnitude of the selective-search contribution to a primacy effect can be directly controlled by manipulating a subject's ability to cognitively reorganize the items in a list. In the absence of extensive, subjective reorganization during rehearsal, subjects may be forced to use other retrieval strategies, such as recalling by a distinctive temporal cue which forms a temporally restricted search set within the list as a whole. On the other hand, Posner (1973) has suggested that there is no compelling reason why parallel memory codes cannot be stored in memory, one temporal and one semantic. Perhaps temporal search will be found to occur to some extent regardless of single-trial subjective reorganization.

\section{REFERENCES}

Atkinson, R. C., \& Shiffrin, R. M. Human memory: A proposed system and its control processes. In $K$. W. Spence \& J. T. Spence (Eds.), The psychology of learning and motivation: Advances in research and theory. Vol. II, New York: Academic Press, 1968.

Bermbach, H. A. Processing strategies for recognition and recall. Joumal of Experimental Psychology, 1973, 99, 409-412

Bjork, R. A., \& Whitten, W. B. Recency sensitive retrieval processes in long-tem free recall. Cognitive Psychology, 1974 $6,173-189$ 
Bruce, D., \& Papay, J. P. Primacy effect in single-trial free recall Joumal of Verbal Learning and Verbal Behavior, 1970, 9 , 473-486.

Carey, S. T., \& Lockhart, R. S。 Encoding differences in recognition and recall. Memory \& Cognition, 1973, 1 , 297-800.

Cicchetti, D. V. Extention of multiple-range tests to interaction tables in the analysis of variance: A rapid approximate solution. Psy chological Bulletin, 1972, 77, 405-408.

Craik, F. I. M., \& Watkins, M. J. The role of rehearsal in short-term memory. Journal of Verbal Leaming and Verbal Behavior, 1973, 12, 599-607.

Glanzer, M. \& Meinzer, A. The effects of intralist activity on free recall. Journal of Verbal Learning and Verbal Behavior, $1967,6,928-935$.

Kay, H. \& Poulton, E. C. Anticipation in memorizing. British Joumal of Psychology, 1951, 41, 34-41.

Loftus, G. R. Recognition and recall in continuous memory. Journal of Experimental Psy chology, 1971, 91, 227-232.

Mandler, G. Organization and recognition. In $E$. Tulving and $W$ Donaldson (Eds.), Organization of memory. New York: Academic Press, 1972.

Norman, D. A., \& Waugh, N. C. Stimulus and response interference in recognition memory experiments. Journal of Experimental Psy chology, 1968, 78, 551-559.
Posner, M. I. Cognition: An introduction. Glenview, lllinois: Scott, Foresman, \& Company, 1973.

Reitman, W., Malin, J. T., Bjork, R. A., \& Higman, B. Strategy control and directed forgetting. Journal of Verbal Learning and Verbal Behavior, 1973, 12, 140-149.

Shiffrin, R. M. Memory search. In D. A. Norman (Ed.), Models of human memory. New York: Academic Press, 1970.

Shiffrin, R. M., \& Atkinson, $R_{\text {. }}$ C. Storage and retrieval processes in long-term memory. Psychological Review, 1969, 76 , 179-193.

Tversky, B. Encoding in recognition and recall. Cognitive Psychology, 1973, 5, 275-287.

Wallace, W. P. Review of the historical, emperical, and theoretical status of the von Restorff phenomenon. Psychological Bulletin, 1965, 63, 410-424.

\section{REFERENCE NOTE}

1. F. S. Bellezza, F. Andrasik, Jr., \& R. P. Lewis. The primacy effect: Rehearsal or search? Paper presented at the meeting of the Midwestern Psychological Association, Chicago, May 1973.

(Received for publication September 15, 1974; accepted October $27,1974$. ) 


\title{
Role selection in the service of self-presentation
}

\author{
MARVIN E. SHAW and PEGGY JO WAGNER \\ University of Florida, Gainesville, Florida 32611
}

\begin{abstract}
Two experiments were conducted to examine the general hypothesis that a person's behavior in a social situation is a function of the kind of impression he wants to make on the other person, and that the kind of impression that he wants to make is influenced by the characteristics of the other person. Two experiments were conducted in which male subjects anticipated interaction with either an attractive female (attractive condition), an unattractive female (unattractive condition), or a female whose attractiveness was unknown (control). Each subject first chose either a high, medium, or low status role to enact during the interaction with the female. He then indicated the role which most closely corresponded to his true role. In Experiment I, role choices were significantly biased in the direction of higher status roles in the attractive condition but not in the unattractive or control conditions. These findings were partially replicated in Experiment II, but difference was shown to be a consequence of the fact that the experimenter was an attractive female. The subjects biased their reports of true role status in the direction of higher status, thus leaving little room for change in the selection of role to be enacted during the social interaction. These findings are consistent with the general hypothesis and with exchange theory predictions.
\end{abstract}

Observers of social interaction have frequently noted that each participant in an interaction engages in behaviors designed to produce an impression upon the other that is in accord with his own interests. Heider (1958) noted that when a person can observe the environment (including other persons), he has more control over it than when he cannot. Knowing this, a given individual may try to influence another's perception of him in order to influence the other's action possibilities, to influence the other's evaluation of him, and/or encourage the other person to communicate or not communicate to others about him. Thus, a person's behavior in social interaction is determined, in part, by the kind of perception that he wants the other person to have of him. Goffman (1959) presents a similar view. He maintained that it is in the person's interest to control the conduct of others, especially their responses to him. "Thus, when an individual appears in the presence of others, there will usually be some reason for him to mobilize his activity so that it will convey an impression to others which it is in his interests to convey (Goffman, 1959, p.4)." Jones (1964) also emphasized the use of self-presentation tactics as a means of gaining favorable social outcomes.

Surprisingly, there is relatively little empirical evidence to support these observations and even less research directed toward the identification of variables determining the kinds of behaviors that are employed in self presentation.

For example, Jones, Gergen, and Jones (1963) reported that high ranking Naval ROTC personnel described themselves more modestly when instructions

The authors wish to thank Robert Lederman and Bill Wienman for serving as experimenters in Experiment $I$, and Susan Wienman and Barbara Cadow for serving as confederates. Requests for reprints should be sent to Marvin E. Shaw, Department of Psychology, University of Florida, Gainesville, Florida 32611. emphasized mutual attraction than when instructions emphasized accuracy, whereas low ranking personnel showed this effect only on "important" items. Gergen and Taylor (1969), again using high and low ranking Naval personnel, found that high status persons described themselves in ways designed to avoid conformity to the expectations of others when productivity was the assigned goal; when the goal was group solidarity, both high and low status persons conformed to the expectations of others. Since status is relative, these studies may reflect self-presentation as a function of the relationship between the person $(P)$ and another (O). However, military rank reflects status relative to all others in the military, and not merely status relative to the particular $O$ with whom $P$ is interacting.

Despite the neglect of the other person in studies of self-presentation, few would disagree that the characteristics of $\mathrm{O}$ influence the kind of impression that $P$ wants to make on 0 , although they may disagree about which characteristics are important and about what effects various characteristics might have on self-presentation. Consider a situation in which a male college student views an attractive or an unattractive female college student through a one-way vision screen, is told that he will hold a conversation with her and that he can choose for enactment one of three roles which reflect either high, medium, or low social status. How will the attractiveness of the female influence the male's choice of roles? Exchange theory (Thibaut \& Kelley, 1959) provides a convenient theoretical framework for analyzing this aspect of dyadic interaction.

The basic assumption of exchange theory is that an individual behaves in ways designed to maximize his reward/cost ratio. In the situation described above, the least costly role to enact is the person's own role or at least a role that is consistent with the person's true 